Ciência Florestal, Santa Maria, v. 20, n. 4, p. 579-588, out.-dez., 2010

ISSN 0103-9954

\title{
COLEÓPTEROS COLETADOS COM ARMADILHAS LUMINOSAS E ETANÓLICAS EM PLANTIO DE Eucalyptus spp. NO SUL DO RIO GRANDE DO SUL
}

\author{
COLEOPTERA COLLECTED WITH LIGHT TRAPS AND ETHANOL TRAPS IN PLANTATION OF \\ Eucalyptus spp. IN THE SOUTH OF THE STATE RIO GRANDE DO SUL
}

Oderlei Bernardi ${ }^{1}$ Mauro Silveira Garcia ${ }^{2}$ Eduardo José Ely e Silva ${ }^{3}$

Luiza Cristiane Fialho Zazycki ${ }^{4}$ Daniel Bernardi ${ }^{5}$ Dinorvan Miorelli ${ }^{6}$

Glodoaldo Arantes Ramiro ${ }^{7}$ Élder Finkenauer ${ }^{8}$

\begin{abstract}
RESUMO
O trabalho teve como objetivo coletar, identificar e estudar a flutuação populacional de espécies da ordem Coleoptera em plantio de Eucalyptus spp. no município de Pinheiro Machado, estado do Rio Grande do Sul. Os insetos foram coletados com armadilhas luminosas e etanólicas a cada quinze dias, no período de fevereiro de 2006 a outubro de 2007. Os exemplares, após triagem, foram identificados por comparação com coleções entomológicas e literatura especializada. Foram coletados 6.172 indivíduos, distribuídos em 39 famílias e 249 espécies, dessas 130 foram identificadas em nível de espécie e 119 em família, representando 4.498 e 1.674 do total de indivíduos coletados respectivamente. As espécies mais abundantes foram Cyclocephala sp. 1, Cyclocephala sp. 2, Dyscinetus sp. 1, Euetheola humilis (Scarabaeidae) e Neoclytus curvatus (Cerambycidae) representando $49,28 \%$ dos indivíduos identificados por gênero e/ou espécie. Scarabaeidae apresentou o maior número de indivíduos (2.588), distribuídos em 37 espécies. As famílias Cerambycidae (47) e Scolytidae (40) apresentaram maior quantidade de espécies. Em todos os meses coletaram-se indivíduos da ordem Coleoptera, com maior número de exemplares em dezembro de 2006 e março de 2007.
\end{abstract}

Palavras-chave: eucalipto; insetos; diversidade; dinâmica populacional.

\begin{abstract}
The objective of this study was to collect, identify and study population fluctuation of Coleoptera species in a forest of Eucalyptus spp., on a farm in the municipality of Pinheiro Machado, Rio Grande do Sul State. Insects were collected with light traps and ethanol traps, once every fifteen days, in the period of February 2006 to October 2007. The insects, after selection procedures, were identified based on entomological

1. Engenheiro Agrônomo, M.Sc., Doutorando em Entomologia pela Escola Superior Agricultura Luiz de Queiroz, Universidade de São Paulo, Av. Pádua Dias 11, Caixa Postal 9, CEP 13418-900, Piracicaba (SP). oderleibernardi@yahoo.com.br

2. Engenheiro Agrônomo, Dr., Professor Associado do Departamento de Fitossanidade, Faculdade de Agronomia Eliseu Maciel, Universidade Federal de Pelotas, Caixa Postal 354, CEP 96010-900, Pelotas (RS). garciasmauro@yahoo.com.br

3. Engenheiro Agrônomo, Dr., Professor do Departamento de Fitossanidade, Faculdade de Agronomia Eliseu Maciel, Universidade Federal de Pelotas, Caixa Postal 354, CEP 96010-900, Pelotas (RS). eduelysilva@uol.com.br

4. Engenheira Agrônoma, Doutoranda em Entomologia pela Escola Superior Agricultura Luiz de Queiroz, Universidade de São Paulo, Av. Pádua Dias 11, Caixa Postal 9, CEP 13418-900, Piracicaba (SP). luizazazycki@gmail.com

5. Engenheiro Agrônomo, Mestrando em Entomologia pela Faculdade de Agronomia Eliseu Maciel, Universidade Federal de Pelotas, Caixa Postal 354, CEP 96010-900, Pelotas (RS).dbernardi2004@yahoo.com.br

6. Engenheiro Agrônomo, Professor do Departamento de Fitossanidade, Faculdade de Agronomia Eliseu Maciel, Universidade Federal de Pelotas, Caixa Postal 354, CEP 96010-900, Pelotas (RS).dm.aratibars@yahoo.com.br

7. Biólogo, Técnico da Empresa Votorantim Celulose \& Papel S/A, Unidade Extremo Sul, BR 116, Km 532, CEP 96160-000, Capão do Leão (RS). glodoaldo.ramiro@vcp.com.br

8. Técnico Agrícola, Técnico da Empresa Votorantim Celulose \& Papel S/A, Unidade Extremo Sul, BR 116, Km 532, CEP 96160-000, Capão do Leão (RS). elder.finkenauer@vcp.com.br

Recebido para publicação em 15/10/2008 e aceito em 11/06/2010
\end{abstract}


Bernardi, O. et al.

collections and specialized literature. A total of 6172 individuals were collected and distributed among 40 families and 249 species, of which 130 were identified at the species level and 119 at the family level, representing 4498 and 1674 of total individuals collected, respectively. Cyclocephala sp. 1, Cyclocephala sp. 2, Dyscinetus sp. 1, Euetheola humilis (Scarabaeidae) and Neoclytus curvatus (Cerambycidae) were the most abundant species, representing $49.28 \%$ of the individuals identified in genus and/or species. Scarabaeidae presented the highest number of individuals (2588), distributed in 37 species. The families Cerambycidae (47) and Scolytidae (40) presented the largest number of species. Individuals of Coleoptera were trapped at all collections but the largest number of individuals was trapped in December 2006 and March 2007.

Keywords: eucalypt; insects; diversity; population dynamics.

\section{INTRODUÇÃO}

No Brasil, espécies do gênero Eucalyptus são plantadas em quase todas as regiões e sua madeira destina-se, especialmente, para a produção de celulose e carvão (IWAKIRI et al., 1999). No sul do Rio Grande do Sul, o cultivo intensificou-se nos últimos anos, como resultado do estabelecimento de empresas do setor madeireiro na região. Atualmente, o Estado ocupa a sexta posição em área cultivada com espécies do gênero Eucalyptus no País com 222.245 ha de um total de 3,7 milhões de hectares (ABRAF, 2008).

As modificações ambientais decorrentes da introdução de monoculturas, incluindo aquelas de eucalipto, podem levar insetos herbívoros a se tornarem pragas nesses ecossistemas. Isso ocorre porque a estrutura da vegetação original determina a distribuição espacial e a disponibilidade de recursos para os herbívoros (SCHOWALTER et al., 1986). Em função disso, as espécies que utilizam o eucalipto como alimento podem ter seu desenvolvimento favorecido e aumentar suas populações, ao contrário as que não o utilizam possivelmente terão suas populações diminuídas, fato que configura uma alteração no equilíbrio populacional anteriormente existente entre as diferentes espécies de insetos que habitavam aquele ambiente.

De acordo com Zanuncio et al. (1994) os danos provocados por insetos fitófagos estão incluídos entre as limitações e as dificuldades para o cultivo de Eucalyptus spp. em grandes áreas de qualquer parte do Brasil. A eucaliptocultura nacional apresenta como principais grupos de insetospraga as formigas, seguido pelos lepidópteros desfolhadores e pelos coleópteros (ANJOS et al., 1986). O último grupo é o menos estudado, apesar de possuir insetos em algumas famílias que podem atacar o eucalipto, como Scolytidae, Cerambycidae, Scarabaeidae, Chrysomelidae, Curculionidae,
Buprestidae e Platypodidae (ZANUNCIO et al., 1993). No entanto, outras famílias como Carabidae, Coccinellidae e Staphylinidae são predadoras (VAN DRIESCHE e BELLOWS, 1996), sendo que as larvas e adultos da primeira família são importantes predadoras de lagartas desfolhadoras e outros insetos (ZANUNCIO et al., 1993).

Nesse sentido, desenvolveu-se um trabalho de coleta, identificação e estudo da flutuação populacional de espécies da Ordem Coleoptera em um plantio de Eucalyptus spp., no município de Pinheiro Machado, estado do Rio Grande do Sul.

\section{MATERIAL E MÉTODOS}

As coletas com armadilhas luminosas e etanólicas foram realizadas em um plantio de Eucalyptus spp. de aproximadamente 2 anos de idade, no período de fevereiro de 2006 a outubro de 2007, em área pertencente à empresa Votorantim Celulose \& Papel S/A, Fazenda São Manoel, localizada às margens da rodovia BR-293 a $7 \mathrm{~km}$ da cidade de Pinheiro Machado, estado do Rio Grande do Sul (31'33'51" S e 5325'59” W) (Figura 1).

Foram utilizadas três armadilhas luminosas de modelo "Al Intral" (FT15T12BL) para a coleta dos coleópteros fototrópicos positivos. As armadilhas foram instaladas a $2 \mathrm{~m}$ do solo, providas de luz negra e acionadas quinzenalmente, durante uma noite, por bateria de 12 volts (ZANUNCIO et al., 1991). Na porção inferior da armadilha, foi fixado um saco plástico com capacidade para 50 litros, contendo no seu interior tiras de papel e no fundo um frasco com algodão embebido em acetato de etila. O objetivo das tiras de papel juntamente com o acetato foi diminuir os danos morfológicos aos exemplares capturados (FERREIRA e MARTINS, 1982).

Foram instaladas também três armadilhas etanólicas de modelo ESALQ-84, confeccionadas

Ci. Fl., v. 20, n. 4, out.-dez., 2010 


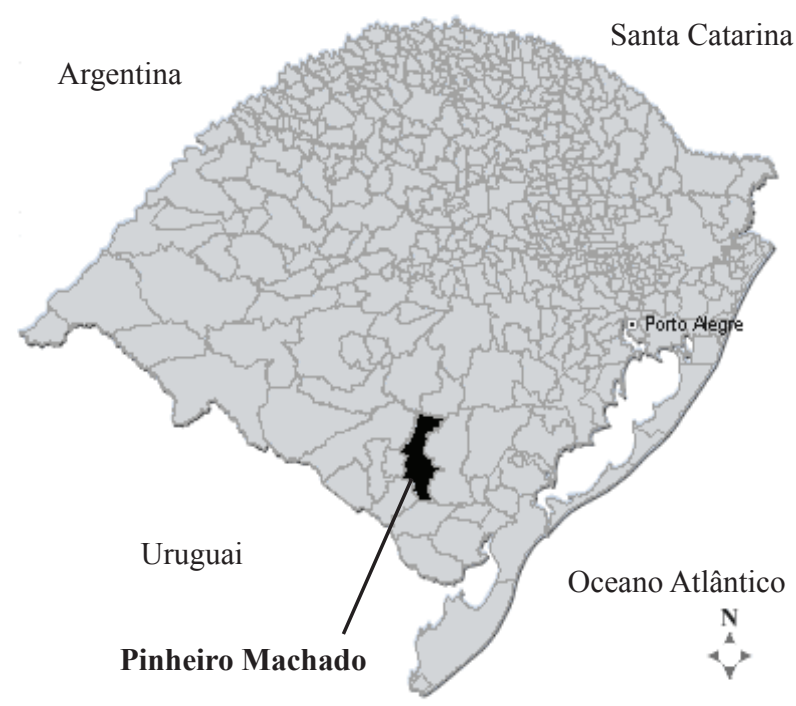

FIGURA 1: Localização geográfica do município de Pinheiro Machado, estado do Rio Grande do Sul, Brasil.

FIGURE 1: Geographical localization of the Municipality of Pinheiro Machado, Rio Grande do Sul State, Brazil.

com chapas de aço galvanizadas de $2 \mathrm{~mm}$ de espessura, a $1,5 \mathrm{~m}$ de altura (FLECHTMANN et al., 1995), tendo como referência a base do cone de captura e iscadas com álcool comercial (96\%), com a função de atrair os insetos. Na porção inferior, foi fixado um frasco de vidro contendo álcool $70 \%$, com a função de coletar os insetos atraídos e interceptados pela armadilha. O período de captura foi quinzenal, quando todos os insetos foram retirados do frasco coletor. Os dados de temperatura e precipitação foram coletados por uma estação meteorológica mantida na área do estudo.

A identificação foi realizada mediante comparação com exemplares do Museu Entomológico "Ceslau Biezanko", Departamento de Fitossanidade, Universidade Federal de Pelotas e por meio de bibliografia especializada (MARTINS, 1999; MARTINS, 2005).

\section{RESULTADOS E DISCUSSÃO}

Foram realizadas 42 coletas, sendo coletados 6.172 indivíduos, distribuídos em 39 famílias e 249 espécies, valores maiores que os encontrados por Pinto et al. (2000), Zanuncio et al. (1993) e Freitas et al. (2002), em trabalhos semelhantes realizados em outras regiões do Brasil.

Das 249 espécies coletadas, 130 foram identificadas em nível de gênero e/ou espécie e 119 identificadas somente por família, perfazendo
72,88 e $27,12 \%$ do total de indivíduos coletados, respectivamente. O baixo número de insetos identificados em nível específico se deve, sobretudo, ao fato de que inexistem especialistas para a identificação de algumas famílias de Coleoptera.

Nos meses de novembro e dezembro de 2006, janeiro, fevereiro e março de 2007 verificouse a maior abundância de coleópteros, com 685,818 , 696, 632 e 988 indivíduos respectivamente, fato que demonstra que esses insetos são mais frequentes nos meses quentes do ano. Já a menor abundância, para os dois anos de coleta, foi observada nos meses de junho, julho, agosto e setembro, quando foram registradas as temperaturas mais baixas (Figura 2).

Cabe ressaltar que a abundância das famílias de Coleoptera pode variar com a região, pois Zanuncio et al. (1993) coletaram maior número de indivíduos nos meses de novembro a abril, nas regiões de São Mateus e Aracruz, estado do Espírito Santo. Pinto et al. (2000) coletaram maior número de coleópteros em setembro na região de Três Marias, estado de Minas Gerais. Oliveira et al. (2001) relataram maior número de coleópteros de setembro a janeiro na região de Nova Era, também em Minas Gerais.

A abundância de espécies de Coleoptera no sul do Rio Grande do Sul sofre influência da temperatura, indicando que esses insetos podem aumentar suas populações em condições ambientais favoráveis e diminuir nas desfavoráveis, tendo essas condições influência direta na emergência dos insetos (Figura 2). Também convém mencionar

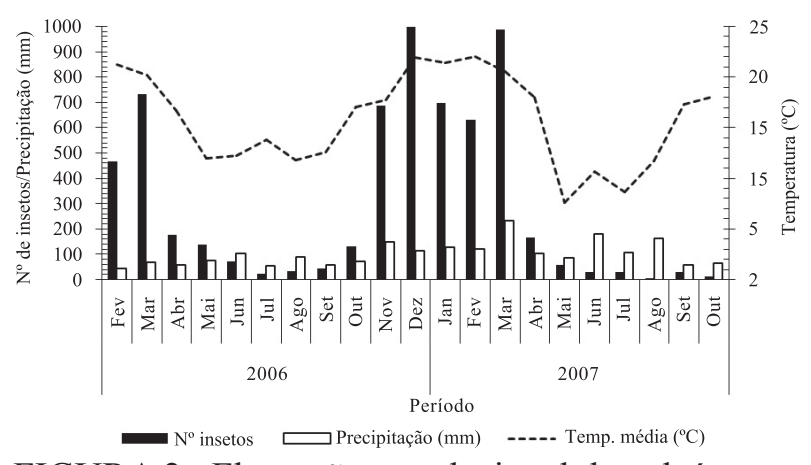

FIGURA 2: Flutuação populacional de coleópteros em plantio de Eucalyptus spp. no município de Pinheiro Machado, estado do Rio Grande do Sul. Fevereiro de 2006 a outubro de 2007.

FIGURE 2: Population fluctuation of beetles in a plantation of Eucalyptus spp. in the Municipality of Pinheiro Machado, Rio Grande do Sul State, Brazil. February 2006 to October 2007. 
que a maioria das espécies coletadas possui ciclo anual sincronizando a emergência nos períodos de temperatura mais propícia, primavera e verão.

As famílias Scarabaeidae, Elateridae, Scolytidae e Cerambycidae com 2.588, 885, 807 e 692 exemplares, respectivamente, apresentaram maior número de indivíduos, perfazendo 80,56\% do total de insetos (Tabela 1). Isso demonstra o potencial e o possível uso das armadilhas luminosas e etanólicas para o monitoramento das espécies dessas famílias em florestamento de eucalipto.

Freitas et al. (2002) também verificaram grande número de Carabidae, Scarabaeidae e Elateridae em coletas com armadilhas luminosas e etanólicas em plantio de Eucalyptus grandis. Mezzomo et al. (1998) relataram que os Scolytidae e Bostrichidae tiveram maior número de indivíduos coletados em armadilhas etanólicas em povoamentos de eucalipto nos municípios de Paineiras e Paraopeba, Minas Gerais. Carvalho (1984) coletou maior número de Scolytidae e Cerambycidae utilizando armadilhas etanólicas e luminosas em plantios de Eucalyptus urophylla e Eucalyptus saligna no município de Salto Alto, estado de São Paulo. Essas constatações demonstram que a diversidade de espécies e o número de indivíduos de Coleoptera, em levantamentos entomofaunísticos, são influenciados por fatores como a região e o tipo da armadilha.

A família Scarabaeidae possui as duas espécies com maior número de indivíduos coletados, Euetheola humilis e Cyclocephala sp. 1 com 967 e 473 indivíduos respectivamente, representando 21,50 e $10,52 \%$ do total de indivíduos identificados por gênero e/ou espécie (Tabela 2). Espécies do gênero Cyclocephala também foram verificadas por Pinto et al. (2000) como as mais abundantes em coletas de coleópteros com armadilhas luminosas em plantio de Eucalyptus urophylla no município de Três Marias, estado de Minas Gerais.

Ocorrendo em densidade populacional elevada o escarabeídeo Euetheola humilis, antes sem registro na eucaliptocultura, ocasionou danos às plantas jovens de eucalipto no município de Candiota, RS, e pode ter se adaptado aos florestamentos de eucalipto no sul do Rio Grande do Sul (BERNARDI et al., 2008). Diante disso, e levando-se em consideração a afirmação de Morón (2004), de que a maior parte das espécies de insetos edafícolas deve estar se adaptando às condições de ecótonos e de agroecossistemas de cultivo, Euetheola humilis pode se estabelecer como praga da eucaliptocultura, o qual deve ter seus danos e população monitorada nos próximos anos para que se possa ter uma melhor avaliação de sua importância para a cultura.

TABELA 1: Número de indivíduos por família de Coleoptera coletados com armadilhas luminosas e etanólicas em plantio de Eucalyptus spp. Pinheiro Machado, Rio Grande do Sul.

TABLE 1: Number of individuals of the Coleoptera collected with light traps and ethanol traps in plantation of Eucalyptus spp.. Pinheiro Machado, Rio Grande do Sul State, Brazil.

\begin{tabular}{lcccc}
\hline \multicolumn{1}{c}{ Famílias } & \multicolumn{4}{c}{ Número de insetos } \\
\cline { 2 - 5 } & 2006 & 2007 & Total & $\%$ \\
\hline Anobiidae & 2 & 0 & 2 & 0,03 \\
Anthicidae & 1 & 0 & 1 & 0,02 \\
Anthribidae & 43 & 16 & 59 & 0,96 \\
Bostrichidae & 83 & 123 & 206 & 3,34 \\
Buprestidae & 1 & 0 & 1 & 0,02 \\
Cantharidae & 36 & 0 & 36 & 0,58 \\
Carabidae & 60 & 141 & 201 & 3,26 \\
Cerambycidae & 410 & 282 & 692 & 11,21 \\
Chrysomelidae & 6 & 0 & 6 & 0,10 \\
Cleridae & 11 & 4 & 15 & 0,24 \\
Coccinellidae & 49 & 31 & 80 & 1,30 \\
Curculionidae & 27 & 4 & 31 & 0,50 \\
Dermestidae & 1 & 0 & 1 & 0,02 \\
Dysticidae & 12 & 0 & 12 & 0,19 \\
Elateridae & 763 & 122 & 885 & 14,34 \\
Endomychidae & 1 & 2 & 3 & 0,05 \\
Erotylidae & 1 & 0 & 1 & 0,02 \\
Geotrupidae & 12 & 5 & 17 & 0,28 \\
Histeridae & 5 & 1 & 6 & 0,10 \\
Hydrophilidae & 2 & 0 & 2 & 0,03 \\
Laemophoeidae & 19 & 1 & 20 & 0,32 \\
Lampyridae & 6 & 0 & 6 & 0,10 \\
Lymexylidae & 1 & 0 & 1 & 0,02 \\
Lucanidae & 3 & 1 & 4 & 0,06 \\
Lyctidae & 1 & 0 & 1 & 0,02 \\
Meloidae & 2 & 1 & 3 & 0,05 \\
Monommidae & 0 & 1 & 1 & 0,02 \\
Nitidulidae & 46 & 9 & 55 & 0,89 \\
Odemenidae & 3 & 0 & 3 & 0,05 \\
Phalacridae & 91 & 0 & 91 & 1,47 \\
Phengodidae & 4 & 52 & 56 & 0,91 \\
Platypodidae & 40 & 77 & 117 & 1,90 \\
Scarabaeidae & 1234 & 1354 & 2588 & 41,93 \\
Scolytidae & 433 & 374 & 807 & 13,08 \\
Silphidae & 26 & 14 & 40 & 0,65 \\
Staphylinidae & 0 & 2 & 2 & 0,03 \\
Tenebrionidae & 26 & 24 & 50 & 0,81 \\
Trogidae & 20 & 3 & 23 & 0,37 \\
Trogossitidae & 43 & 4 & 47 & 0,76 \\
\hline Total & & & & \\
\hline & & 2.648 & 172 & 100,00 \\
\hline
\end{tabular}


TABELA 2: Número de indivíduos por espécie de Coleoptera coletados com armadilhas luminosas e etanólicas em plantio de Eucalyptus spp. no município de Pinheiro Machado, Rio Grande do Sul. Fevereiro de 2006 à outubro de 2007.

TABLE 2: Number of individuals for species of Coleoptera collected with light traps and ethanol traps in plantation of Eucalyptus spp. in the Municipal district of Pinheiro Machado, Rio Grande do Sul State, Brazil. February 2006 to October 2007.

\begin{tabular}{|c|c|c|}
\hline Famílias/Espécies & Total & $\%$ \\
\hline Carabidae & 4 & 0,09 \\
\hline Enceladus gigas Bonelli, 1813 & 23 & 0,51 \\
\hline Scarites sp. & 15 & 0,33 \\
\hline \multicolumn{3}{|l|}{ Cerambycidae } \\
\hline Acanthoderes jaspidea (Germar, 1824) & 23 & 0,51 \\
\hline Acanthoderes nigricans Lameere, 1885 & 3 & 0,07 \\
\hline Achryson surinamum (Linnaeus, 1767) & 3 & 0,07 \\
\hline Aerenea sp. & 1 & 0,02 \\
\hline Centrocerum elegans (Chevrolat, 1861) & 3 & 0,07 \\
\hline Chlorida costata Audinet-Seville, 1834 & 23 & 0,51 \\
\hline Chlorida festiva Linnaeus, 1758 & 7 & 0,16 \\
\hline Chydarteres striatus striatus (Fabricius, 1787) & 8 & 0,18 \\
\hline Compsoceridius gounellei (Bruch, 1908) & 1 & 0,02 \\
\hline Compsocerus barbicornis Audinet - Serville, 1834 & 47 & 1,04 \\
\hline Compsocerus violaceus (White, 1853) & 3 & 0,07 \\
\hline Desmiphora sp. & 1 & 0,02 \\
\hline Dorcadocerus barbatus (Olivier, 1790) & 1 & 0,02 \\
\hline Eburodacris sp. & 20 & 0,44 \\
\hline Erosida lineola (Fabricius, 1781) & 2 & 0,04 \\
\hline Eurysthea hirta (Kirby, 1818) & 44 & 0,98 \\
\hline Eurysthea lacordairei (Lacordaire, 1869) & 1 & 0,02 \\
\hline Hippopsis sp. & 1 & 0,02 \\
\hline Hyperplatys argentinus (Berg, 1889) & 2 & 0,04 \\
\hline Leptostylus obscurellus Bates, 1863 & 2 & 0,04 \\
\hline Lypsimena nodipennis (Burmeister, 1865) & 4 & 0,09 \\
\hline Martinsellus signatus (Gyllenhal, 1817) & 1 & 0,02 \\
\hline Megacyllene acuta (Germar, 1821) & 5 & 0,11 \\
\hline Nealcidion bicristatum (Bates, 1863) & 2 & 0,04 \\
\hline Neoclytus centurio (Chevrolat, 1862) & 17 & 0,38 \\
\hline Neoclytus curvatus (Germar, 1821) & 281 & 6,25 \\
\hline Neoclytus pusillus (Laporte \& Gory, 1836) & 16 & 0,36 \\
\hline Neoclytus ypsilon Chevrolat, 1865 & 15 & 0,33 \\
\hline Neodillonia adspersa (Germar, 1824) & 1 & 0,02 \\
\hline Nyssodrisina lignaria (Bates, 1863) & 107 & 2,38 \\
\hline Oncideres saga Dalman (1823) & 1 & 0,02 \\
\hline Oreodera remota (Pascoal, 1859) & 1 & 0,02 \\
\hline Oxymerus luteus luteus (Voet,1778) & 3 & 0,07 \\
\hline Paramoecerus barbicornis (Fabricius, 1792) & 13 & 0,29 \\
\hline Phoracantha recurva Newman, 1840 & 1 & 0,02 \\
\hline Praxithea derourei (Charillae, 1857) & 1 & 0,02 \\
\hline Retrachyderes thoraxicus (Olivier,1790) & 13 & 0,29 \\
\hline Thelgetra latipennis Thomson, 1864 & 1 & 0,02 \\
\hline Unxia gracilior (Burmeister, 1865) & 2 & 0,04 \\
\hline \multicolumn{3}{|l|}{ Chrysomelidae } \\
\hline Cerotoma sp. & 1 & 0,02 \\
\hline \multicolumn{3}{|l|}{ Coccinellidae } \\
\hline Cycloneda callispilota (Guérin, 1844) & 4 & 0,09 \\
\hline Cycloneda sanguinea (Linnaeus, 1763) & 5 & 0,11 \\
\hline Cycloneda sp. & 4 & 0,09 \\
\hline Harmonia axyridis (Pallas, 1773) & 56 & 1,24 \\
\hline
\end{tabular}

Continua... 
TABELA 2: Continuação...

TABLE 2: Continued...

\begin{tabular}{|c|c|c|}
\hline Famílias/Espécies & Total & $\%$ \\
\hline Olla sp. & 2 & 0,04 \\
\hline Olla v-nigrum Mulsant, 1866 & 4 & 0,09 \\
\hline \multicolumn{3}{|l|}{ Curculionidae } \\
\hline Panthomorus sp. & 1 & 0,02 \\
\hline Elateridae & & 0,00 \\
\hline Conoderus sp. 1 & 110 & 2,45 \\
\hline Conoderus sp. 2 & 142 & 3,16 \\
\hline Conoderus sp. 3 & 122 & 2,71 \\
\hline Conoderus sp. 4 & 20 & 0,44 \\
\hline Conoderus sp. 5 & 5 & 0,11 \\
\hline Photinus sp. & 14 & 0,31 \\
\hline Meloidae & & 0,00 \\
\hline Epicauta atomaria (Germar, 1821) & 3 & 0,07 \\
\hline Platypodidae & & 0,00 \\
\hline Euplatypus pulicans (Chapuis) & 7 & 0,16 \\
\hline Tesserocerus elegans Chapuis & 32 & 0,71 \\
\hline Tesserocerus insignis Saunders, 1936 & 42 & 0,93 \\
\hline \multicolumn{3}{|l|}{ Scarabaeidae } \\
\hline Agocephala sp. & 27 & 0,60 \\
\hline Anomala sp. 1 & 9 & 0,20 \\
\hline Anomala sp. 2 & 3 & 0,07 \\
\hline Anomala sp. 3 & 1 & 0,02 \\
\hline Bothynus sp. 1 & 22 & 0,49 \\
\hline Bothynus sp. 2 & 7 & 0,16 \\
\hline Canthon sp. & 2 & 0,04 \\
\hline Chalepides barbata Fabricius, 1787 & 6 & 0,13 \\
\hline Cyclocephala sp. 1 & 473 & 10,52 \\
\hline Cyclocephala sp. 2 & 232 & 5,16 \\
\hline Diloboderus abderus Sturm, 1826 & 1 & 0,02 \\
\hline Diplotaxis $\mathrm{sp}$. & 2 & 0,04 \\
\hline Dyscinetus sp. 1 & 263 & 5,85 \\
\hline Dyscinetus sp. 2 & 35 & 0,78 \\
\hline Dyscinetus sp. 3 & 2 & 0,04 \\
\hline Euetheola humilis Burmeister, 1847 & 967 & 21,50 \\
\hline Euetheola sp. & 2 & 0,04 \\
\hline Euphoria lurida (Fabricius, 1775) & 22 & 0,49 \\
\hline Geniates sp. & 11 & 0,24 \\
\hline Hemiphileurus sp. & 1 & 0,02 \\
\hline Isonychus sp. 2 & 145 & 3,22 \\
\hline Leucothyreus femortus Burmeister, 1844 & 24 & 0,53 \\
\hline Leucothyreus sp. & 8 & 0,18 \\
\hline Lygirus sp. & 11 & 0,24 \\
\hline Pelidnota aeruginosa (Linnaeus, 1758) & 1 & 0,02 \\
\hline Phileurus sp. & 4 & 0,09 \\
\hline Phyllophaga sp. 1 & 1 & 0,02 \\
\hline Phylophaga sp. 2 & 1 & 0,02 \\
\hline Pinotus sp. & 5 & 0,11 \\
\hline \multicolumn{3}{|l|}{ Scolytidae } \\
\hline Ambrosiodmus obliquus LeConte, 1878 & 3 & 0,07 \\
\hline Ambrosiodmus sp. & 3 & 0,07 \\
\hline Corthylus antennarius Schedl, 1966 & 85 & 1,89 \\
\hline Corthylus comitabilis Wood & 2 & 0,04 \\
\hline Corthylus gracillens Wood & 6 & 0,13 \\
\hline Corthylus nudipennis Schedl, 1950 & 4 & 0,09 \\
\hline Corthylus punctatus Zimmermann, 1868 & 10 & 0,22 \\
\hline
\end{tabular}

Continua...

Ci. Fl., v. 20, n. 4, out.-dez., 2010 
TABELA 2: Continuação...

TABLE 2: Continued...

\begin{tabular}{|c|c|c|}
\hline Famílias/Espécies & Total & $\%$ \\
\hline Corthylus sp. & 16 & 0,36 \\
\hline Cryptocarenus brevicollis Wood & 6 & 0,13 \\
\hline Cryptocarenus diadematus Eggers, 1937 & 2 & 0,04 \\
\hline Cryptocarenus sp. & 1 & 0,02 \\
\hline Dryocoetoides asperulus (Eggers, 1931) & 1 & 0,02 \\
\hline Hylocurus dimorphus (Schedl) & 2 & 0,04 \\
\hline Hyphotenemus eruditus Westwood, 1836 & 13 & 0,29 \\
\hline Hyphotenemus sp. & 1 & 0,02 \\
\hline Hypothenemus aff. dolosus Wood & 2 & 0,04 \\
\hline Hypothenemus bolivianus Eggers, 1931 & 1 & 0,02 \\
\hline Microcorthylus minimus Schedl, 1950 & 13 & 0,29 \\
\hline Microcorthylus quadridens Wood & 13 & 0,29 \\
\hline Microcorthylus sp. & 6 & 0,13 \\
\hline Monarthrum brasiliensis (Schedl, 1936) & 8 & 0,18 \\
\hline Monarthrum cristatus Blandford, 1905 & 7 & 0,16 \\
\hline Monarthrum sp. & 3 & 0,07 \\
\hline Tricolus bifidus Schedl,1939 & 8 & 0,18 \\
\hline Tricolus sphenicus Schedl, 1939 & 1 & 0,02 \\
\hline Xyleborinus saxeseni (Ratzeburg, 1837) & 6 & 0,13 \\
\hline Xyleborinus sp. & 17 & 0,38 \\
\hline Xyleborus affinis Eichhoff, 1867 & 2 & 0,04 \\
\hline Xyleborus alter Eggers, 1931 & 3 & 0,07 \\
\hline Xyleborus biconicus Eggers, 1928 & 2 & 0,04 \\
\hline Xyleborus ferrugineus (Fabricius, 1801) & 72 & 1,60 \\
\hline Xyleborus gracilis Eichhoff, 1868 & 11 & 0,24 \\
\hline Xyleborus linearicollis Schedl, 1937 & 102 & 2,27 \\
\hline Xyleborus neivai Eggers, 1928 & 1 & 0,02 \\
\hline Xyleborus posticus Eichhoff, 1868 & 1 & 0,02 \\
\hline Xyleborus retusus Eichhoff, 1868 & 202 & 4,49 \\
\hline Xyleborus sentosus Eichhoff, 1868 & 12 & 0,27 \\
\hline \multicolumn{3}{|l|}{ Silphidae } \\
\hline Sylpha sp. & 40 & 0,89 \\
\hline \multicolumn{3}{|l|}{ Trogidae } \\
\hline Omargus sp. 1 & 5 & 0,11 \\
\hline Omargus sp. 2 & 10 & 0,22 \\
\hline Omargus sp. 3 & 9 & 0,20 \\
\hline Total & 4.498 & 100 \\
\hline
\end{tabular}

A família Elateridae com maior abundância em novembro e dezembro com 289 e 380 indivíduos, respectivamente (Figura 3), apresenta espécies importantes para a eucaliptocultura, pois são predadores de pragas dessa cultura, durante sua fase jovem ou adulta (ZANUNCIO et al., 1993). No entanto, praticamente todos os insetos coletados pertencem ao gênero Conoderus cujas larvas e adultos alimentam-se principalmente de tecidos vegetais.

A família Scolytidae apresentou 807 indivíduos, distribuídos em quarenta espécies (Tabela 2), sendo estas coletadas durante os meses de temperatura mais elevada (Figura 4). $\mathrm{O}$ gênero Xyleborus com nove espécies é responsável por $50,56 \%$ do total de Scolytidae, sendo a espécie
Xyleborus retusus com 202 indivíduos a mais abundante (Tabela 2).

De acordo com Samaniego e Gara (1970) durante os meses mais frios, a temperatura média em torno de $15^{\circ} \mathrm{C}$ não é adequada ao vôo de Scolytidae. Para o caso específico do gênero Xyleborus os mesmos autores verificaram que a temperatura de $21^{\circ} \mathrm{C}$ é o limiar mínimo para que as espécies iniciem seu vôo. Fato que explica em parte a maior quantidade de indivíduos capturados nas estações quentes, bem como, possivelmente, por essas espécies possuírem ciclo anual, ocorra a emergência dos adultos nos períodos de temperatura mais propícia para o seu desenvolvimento e reprodução, o que contribui para uma maior abundância desses insetos nos períodos de temperatura mais elevada. 
Bernardi, O. et al.

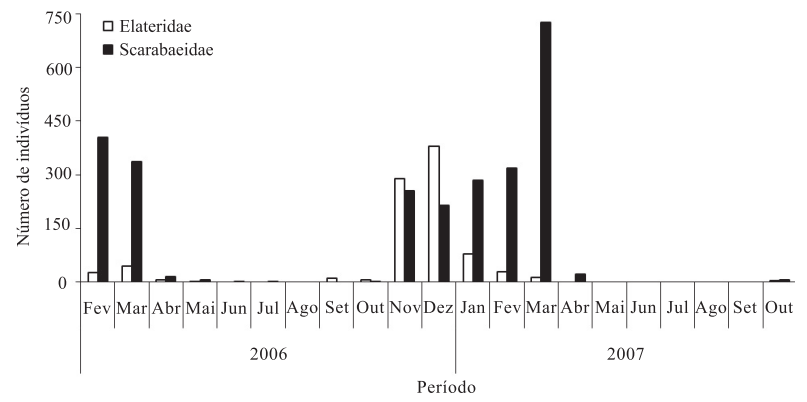

FIGURA 3: Número de indivíduos de Elateridae e Scarabaeidae coletados com armadilhas luminosas e etanólicas em plantio de Eucalyptus spp. no município de Pinheiro Machado, estado do Rio Grande do Sul. Fevereiro de 2006 a outubro de 2007.

FIGURE 3: Number of individuals of Elateridae and Scarabaeidae collected with light traps and ethanol traps in plantation of Eucalyptus spp. in the Municipality of Pinheiro Machado, Rio Grande do Sul State, Brazil. February 2006 to October 2007.

A família Cerambycidae foi coletada frequentemente, sendo registrado grande número de indivíduos (692) e espécies (47), basicamente coletados nos meses de temperaturas mais elevadas (Figura 4). Essa famíliajá foi observadapor Mezzomo et al. (1998) como uma das mais abundantes em povoamentos de eucalipto no município Paraopeba, estado de Minas Gerais. Já Carvalho (1984) coletou maior número de Scolytidae e Cerambycidae em povoamentos de Eucalyptus urophylla e Eucalyptus saligna no município de Salto, estado de São Paulo.

A verificação de grande quantidade de espécies e indivíduos de Scolytidae em plantio jovem de eucalipto é contrária à suposição de Disperati (1995), segundo o qual ocorreria pequena quantidade de Scolytidae em povoamentos jovens de eucaliptos e menores abundâncias em povoamentos com idades mais avançadas, porque o vigor e a capacidade de defesa desses povoamentos aumentam com o avanço da idade. No entanto, a elevada abundância desses insetos no florestamento de eucalipto pode ser por causa da coleta de indivíduos oriundos das áreas de preservação permanente como mata nativa, que remanesce entre os talhões, mas também pode ser pelos insetos encontrarem no eucalipto uma fonte de alimento e local de reprodução.

Dentre as diversas espécies de Cerambycidae, as pertencentes ao gênero Neoclytus são responsáveis por $47,54 \%$ do total de indivíduos dessa família e a espécie Neoclytus curvatus a mais abundante com 281 indivíduos (Tabela 2). Segundo Zanuncio et al. (1993), insetos do gênero Neoclytus possuem grande capacidade de dano a muitas espécies arbóreas, dentre elas o eucalipto, os quais se caracterizam pela abertura de galerias no tronco das árvores. Neoclytus curvatus já foi citada por Monné et al. (2002) ocasionando danos às plantas de Eucalyptus grandis e Eucalyptus globulus na Província de Lavalleja no Uruguai. Outra espécie do gênero verificada nas coletas é Neoclytus pussilus a qual é citada no Brasil por Zanuncio et al. (1993), como potente broqueadora de troncos de Eucalyptus pellita, Eucalyptus tereticornis e Eucalyptus urophylla.

Das famílias de Coleoptera amostradas, Carabidae eCoccinelidae são importantes predadores de lagartas e ovos de lepidópteros desfolhadores, mas ocorreram em pequeno número com $201 \mathrm{e}$ 80 indivíduos respectivamente. Em áreas onde o cultivo de eucalipto é realizado há mais tempo, os Carabidae têm ocorrido em grande número, conforme demonstrado por Pinto et al. (2000), em levantamento de coleópteros em reflorestamento de Eucalyptus urophylla em Três Marias, estado de Minas Gerais onde essa família apresentou o maior

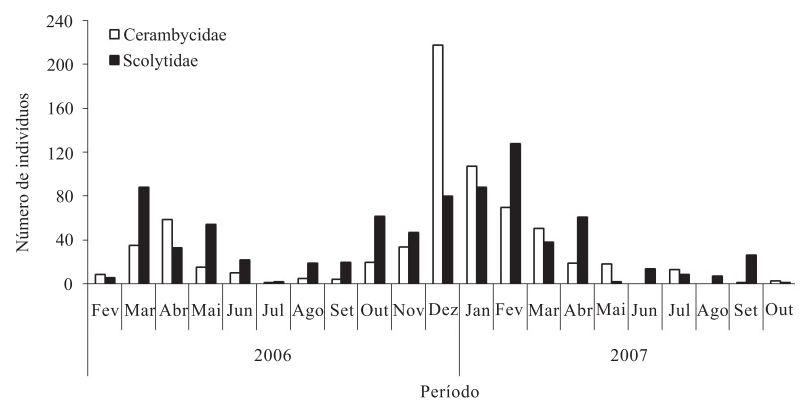

FIGURA 4: Número de indivíduos de Cerambycidae e Scolytidae coletados com armadilhas luminosas e etanólicas em plantio de Eucalyptus spp. no município de Pinheiro Machado, estado do Rio Grande do Sul. Fevereiro de 2006 a outubro de 2007.

FIGURE 4: Numberofindividuals ofCerambycidae and Scolytidae collected with light traps and ethanol traps in plantation of Eucalyptus spp. in the Municipality of Pinheiro Machado, Rio Grande do Sul State, Brazil. February 2006 to October 2007. 
número de indivíduos, representando $95,13 \%$ do total de coleópteros.

Freitas et al. (2002) verificaram, em plantio de Eucalyptus grandis na região de Santa Bárbara, Minas Gerais, que os Carabidae com 3.560 indivíduos, representaram $74,55 \%$ enquanto que os Coccinelidae com 26 indivíduos representaram apenas $0,54 \%$ dos insetos coletados com armadilhas luminosas. Cabe ressaltar que, caso ocorra implantação de algum programa de monitoramento de insetos-praga na região, deve-se utilizar armadilhas luminosas com marcador de tempo conforme recomendam Zanuncio et al. (1993), para que estas funcionem fora dos períodos de vôo de espécies de Carabidae, para evitar reduzir o número de indivíduos capturados dessa família.

As famílias Anobiidae, Anthicidae, Buprestidae, Chrysomelidae, Dermestidae, Erotylidae, Histeridae, Hydrophilidae, Lampyridae, Limexylidae, Lyctidae, Lucanidae, Meloidae, Monommidae, Odemenidae e Stahphylinidae apresentaram pequena abundância de indivíduos durante todo o período de amostragem (Tabela 1). Todavia, insetos pouco abundantes ou raros de acordo com Delabie et al. (2000), desempenham funções indiretas e em casos nos quais ocorram alterações nas comunidades, podem também se adaptar ao novo meio e manter a diversidade na comunidade.

Cabe ressaltar que de acordo com Morales et al. (1999), estudos de levantamento populacional demonstram apenas a abundância das diferentes espécies em determinado ambiente ou ecossistema e geram poucas informações a respeito do ciclo, sazonalidade e dinâmica populacional ao longo do tempo. Nesse sentido, no Sul do Rio Grande do Sul, existe a necessidade de monitoramento, no decorrer dos anos de cultivo, daquelas espécies com potencial de atacarem a cultura do eucalipto.

\section{CONCLUSÕES}

O florestamento de eucalipto estudado apresenta elevada diversidade de espécies de Coleoptera. Caso ocorra a implementação de programas de monitoramento de insetos com o uso de armadilhas luminosas e etanólicas em florestamentos de eucalipto da região, este deve ser preferencialmente realizado nas épocas mais quentes do ano.

As espécies mais abundantes de coleópteros são Cyclocephala sp. 1, Cyclocephala sp. 2,
Dyscinetus sp. 1, Euetheola humilis e Neoclytus curvatus.

Scarabaeidae apresenta maior número de indivíduos coletados, e Cerambycidae e Scolytidae maior número de espécies.

Até o momento, dentre as diferentes espécies de Coleoptera coletadas, foram constatados ataques somente de Euetheola humilis à plantas de eucalipto no sul do Rio Grande do Sul.

\section{AGRADECIMENTOS}

Ao taxonomista Dr. Eduardo José Ely e Silva do Museu Entomológico "Ceslau Biezanko", Departamento de Fitossanidade, Universidade Federal de Pelotas e aos especialistas PhD. Carlos Alberto Hector Flechtmann, Universidade Estadual Paulista "Júlio de Mesquita Filho" (UNESP), Campus de Ilha Solteira e ao Dr. Sérgio Ide, Instituto Biológico, Universidade de São Paulo, pela ajuda concedida para a identificação dos insetos.

\section{REFERÊNCIAS BIBLIOGRÁFICAS}

ABRAF. Associação brasileira de produtores de florestas plantadas. Anuário estatístico 2008 (Ano base 2007). 2008. Disponível em: <(http://www. abraflor.org.br/estatística.asp)> Acesso em: 28 de março de 2009.

ANJOS, N.; SANTOS, G. P.; ZANUNCIO, J. C. Pragas do eucalipto e seu controle. Informe Agropecuário, Belo Horizonte, v. 12, n. 141, p. 5158, set./out. 1986.

BERNARDI, O. et al. Ocorrência de Euetheola humilis (Burmeister) (Coleoptera: Scarabaeidae) em Eucalyptus saligna Smith (Myrtaceae), no Estado do Rio Grande do Sul. Neotropical Entomology, Londrina, v. 37, n. 1, p. 100-103, jan./fev. 2008.

CARVAlHO, A. O. R. Análise faunística de coleópteros coletados em plantas de Eucalyptus urophylla S.T. Blake e Eucalyptus saligna Sm. 1984. 105 f. Dissertação (Mestrado em Ciências/ Entomologia)-Universidade de São Paulo. Escola Superior de Agricultura "Luiz de Queiroz", Piracicaba, 1984.

DELABIE, J. H. C; FRESNEAU, D.; PEZON, A. Notes on the ecology of Thaumatomyrmex spp. (Hymenoptera: Formicidae: Ponerinae) in southeast Bahia, Brazil. Sociobiology, Califórnia, v. 36, n. 3, p. 571-584, jan./fev. 2000.

DISPERATI, A. A. Sensoriamento remoto para a deteç̧ão, mapeamento e monitoramento dos 
danos causados por pragas florestais. Curitiba, Brasil: FUPEF, 1995. 40 p.

FERREIRA, P. S. F.; MARTINS, D. S. Contribuição ao método de captura de insetos por meio de armadilha luminosa, para a obtenção de exemplares sem danos morfológicos. Revista Ceres, Viçosa, v. 29, n. 165, p. 538-543, nov./dez. 1982.

FLECHTMANN, C. A. H. et al. Manual de pragas em florestas - Scolytidae em reflorestamento com pinheiros tropicais. Piracicaba, Brasil: Programa Cooperativo de Manejo de Pragas Florestais - IPEF, 1995. $201 \mathrm{p}$

FREITAS, F. A. et al. Fauna de Coleoptera coletada com armadilhas luminosas em um plantio de Eucalyptus grandis em Santa Bárbara, Minas Gerais. Revista Árvore, Viçosa, v. 26, n. 4, p. 505 511, jul./ago. 2002.

IWAKIRI, S.; PEREIRA, S. J.; NISGOSKI, S. Avaliação da qualidade de colagem em compensados de Eucalyptus cloeziana e Eucalyptus robusta. Floresta e Ambiente, Rio de Janeiro, v. 6, n. 1, p. 45-50, jan./dez. 1999.

MARTINS, U. R. Cerambycidae Sul-Americanos (Coleoptera) - III. Subfamíia Cerambycinae, Hesperophanini Mulsant, 1839; Eburiini Blanchard, 1845; Diorini Lane, 1950. São Paulo: EdUSP, 1999. 418 p.

MARTINS, U. R. Cerambycidae Sul-Americanos (Coleoptera) - VII. Subfamília Cerambycinae, Elaphidionini Thamson 1864. São Paulo: EdUSP, 2005. 394 p.

MEZZOMO, J. A. et al. Influência de faixas de vegetação nativa sobre Coleoptera em Eucalyptus cloeziana. Revista Árvore, Viçosa, v. 22, n. 1, p. 77-87, jan./fev. 1998.

MORÓN, M. A. Melolontídeos edafícolas. In: SALVADORI, J. R.; ÁVILA, C. J.; SILVA, M. T. B (Eds). Pragas de solo no Brasil. Passo Fundo: Embrapa Trigo. Dourados: Embrapa Agropecuária Oeste. Cruz Alta: Fundacep Fecotrigo, 2004. p.133 166.

MORALES, N. E. et al. Índices populacionais de besouros Scolytidae em reflorestamento de Eucalyptus grandis W. Hill ex. Maiden no município de Antônio Dias, Minas Gerais. Revista Árvore, Viçosa, v. 22, n. 3, p. 359-363, maio/jun. 1999.

MONNÉ, M. A. et al. Cerambicídeos (Coleoptera) que atacam Eucalyptus globulos e Eucalyptus grandis en Uruguay. Agrociencia, Coyoacán, v. 6, n. 1, p. 63-68, jan./fev. 2002.

OLIVEIRA, H. G. et al. Flutuação populacional de coleópteros associados a eucaliptocultura na região de Nova Era, Minas Gerais. Floresta e Ambiente, Rio de Janeiro, v. 8, n. 1, p. 52-60, jan./dez. 2001.

PINTO, R. et al. Flutuação populacional de Coleoptera em plantio de Eucalyptus urophylla no Município de Três Marias, Minas Gerais. Floresta e Ambiente, Rio de Janeiro, v. 7, n. 1, p. 143-151, jan./dez. 2000.

SAMANIEGO, A.; GARA, R. I. Estudios sobre la actividad de vuelo y selección de huéspedes por Xyleborus spp. y Platypus spp. (Coleoptera: Scolytidae y Platypodidae). Turrialba, San José, v. 20, n. 2, p. 471-477, jul./dez. 1970.

SCHOWALTER, T. D.; HARGROVE, W. W.; CROSSLEY JR., D. A. Herbivory in forested ecosystems. Annual Review of Entomology, Palo Alto, v. 31, p. 177-196, jan./dez. 1986.

VAN DRIESCHE, R. G.; BELLOWS JR., T. S. Biological control. New York: Chapman \& Hall, 1996. $539 \mathrm{p}$.

ZANUNCIO, J. C. et al. Major lepidopterous defoliators of eucalypt in southeast Brazil. Forest Ecology and Management, Amsterdam, v. 65, p. 53-63, maio. 1994.

ZANUNCIO, J. C. et al. Coleópteros associados à eucaliptocultura nas regiões de São Mateus e Aracruz, Espírito Santo. Revista Ceres, Viçosa, v. 41, n. 232, p. 584-590, nov./dez. 1993.

ZANUNCIO, J. C. et al. Levantamento e flutuação populacional de lepidópteros associados a eucaliptocultura: II- Região do Alto São Francisco, MG. Anais da Sociedade Entomológica do Brasil, Londrina, v. 20, n. 2, p. 285-292, mar./abr. 1991. 